\title{
Independent genome-wide scans identify a chromosome 18 quantitative-trait locus influencing dyslexia
}

\author{
Simon E. Fisher ${ }^{1 \star}$, Clyde Francks ${ }^{1 \star}$, Angela J. Marlow ${ }^{1}$, I. Laurence MacPhie ${ }^{1}$, Dianne F. Newbury ${ }^{1}$, Lon R. \\ Cardon $^{1}$, Yumiko Ishikawa-Brush ${ }^{1}$, Alex J. Richardson ${ }^{2}$, Joel B. Talcott ${ }^{2}$, Javier Gayán ${ }^{3}$, Richard K. Olson ${ }^{3}$, \\ Bruce F. Pennington ${ }^{4}$, Shelley D. Smith ${ }^{5}$, John C. DeFries ${ }^{3}$, John F. Stein ${ }^{2} \&$ Anthony P. Monaco ${ }^{1}$ \\ ${ }^{*}$ These authors contributed equally to this work.
}

Published online: 17 December 2001, DOI: 10.1038/ng792

Developmental dyslexia is defined as a specific and significant impairment in reading ability that cannot be explained by deficits in intelligence, learning opportunity, motivation or sensory acuity. It is one of the most frequently diagnosed disorders in childhood, representing a major educational and social problem ${ }^{1}$. It is well established that dyslexia is a significantly heritable trait $^{2}$ with a neurobiological basis ${ }^{3}$. The etiological mechanisms remain elusive, however, despite being the focus of intensive multidisciplinary research ${ }^{3}$. All attempts to map quantitative-trait loci (QTLs) influencing dyslexia susceptibility have targeted specific chromosomal regions, so that inferences regarding genetic etiology have been made on the basis of very limited information ${ }^{4}$. Here we present the first two complete QTL-based genome-wide scans for this trait, in large samples of families from the United Kingdom and United States. Using single-point analysis, linkage to marker D18S53 was independently identified as being one of the most significant results of the genome in each scan $(P \leq 0.0004$ for single word-reading ability in each family sample). Multipoint analysis gave increased evidence of 18p11.2 linkage for single-word reading, yielding top empirical $P$ values of 0.00001 (UK) and 0.0004 (US). Measures related to phonological and orthographic processing also showed linkage at this locus. We replicated linkage to 18 p11.2 in a third independent sample of families (from the UK), in which the strongest evidence came from a phoneme-awareness measure (most significant $P$ value $=0.00004$ ). A combined analysis of all UK families confirmed that this newly discovered $18 p$ QTL is probably a general risk factor for dyslexia, influencing several readingrelated processes. This is the first report of QTL-based genomewide scanning for a human cognitive trait.

A large body of evidence accumulated from many family- and twin-based studies over the past century indicates that genetic factors have a significant role in predisposition towards developmental dyslexia ${ }^{4}$. The power of gene-mapping studies is limited, however, by the genetic complexity of the trait, in which factors such as incomplete penetrance, phenocopies, heterogeneity and oligogenicity are likely to be involved ${ }^{4}$. In addition, there can be considerable phenotypic variability among individuals classified
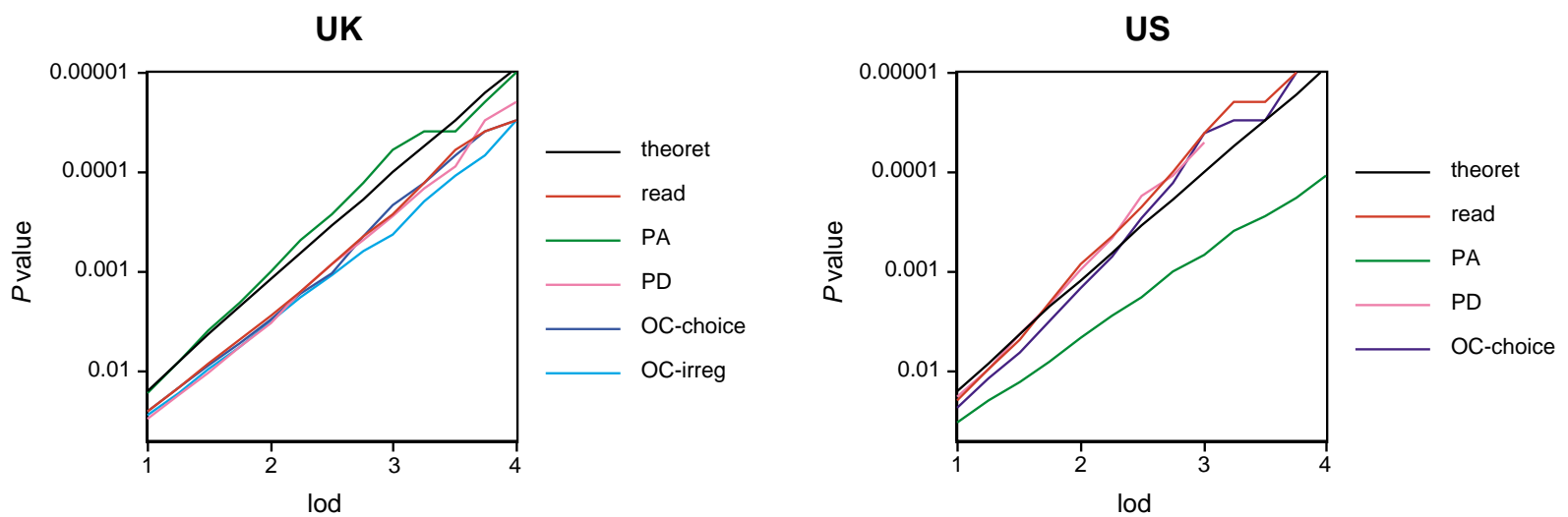

Fig. 1 Relationship between VC lod scores and pointwise significance of linkage for different measures in each genome-screen sample. We determined these results empirically from 100,000 replicate simulations assuming $75 \%$ heterozygosity (Methods). Comparison with chromosome 18 -specific simulations indicated that these distributions were valid for determining empirical pointwise significance from the multipoint genome-scan data (Methods). $P$ values from the simulated distributions can be compared with the theoretical asymptotic estimates of significance for each lod score (calculated by multiplying by 2 log $_{\mathrm{e}} 10$ and looking for the result in $\chi^{2}$ tables $^{20}$ ). These results show that in real family samples, although some measures behave similarly to what theory would predict, others may suffer from an inflation of the type I error rate (as suggested in ref. 19). Estimating significance from the simulation data for each measure allows us to control appropriately for this problem when interpreting genome-scan results. The relationship between the lod scores and empirical significance can differ substantially between family samples. The PA measure in the UK sample, for example, behaves as predicted by theory, whereas a comparable measure in the US sample gives an inflated type I error. Observations such as this may be partly caused by differences in ascertainment between the samples.

${ }^{1}$ Wellcome Trust Centre for Human Genetics, University of Oxford, Roosevelt Drive, Oxford, OX3 7BN, UK. ${ }^{2}$ Department of Physiology, University of Oxford, Parks Road, Oxford, OX1 3PT, UK. ${ }^{3}$ Institute for Behavioral Genetics, University of Colorado, Boulder, Colorado 80309-0447, USA. ${ }^{4}$ Department of Psychology, University of Denver, Denver, Colorado 80208, USA. ${ }^{5}$ Center for Human Molecular Genetics, University of Nebraska Medical Center, Omaha, Nebraska 68198-5455, USA. Correspondence should be addressed to A.P.M. (e-mail: anthony.monaco@well.ox.ac.uk). 
as dyslexic, which further complicates genetic analysis. These problems can be overcome to a certain extent by adopting QTL strategies that directly investigate linkage to quantitative measures of reading-related processes in sibling pairs ${ }^{5-8}$ rather than relying on categorical diagnostic criteria. In recent years, there have been several focused investigations, using both qualitative and quantitative phenotypes, of the role of particular chromosomal regions (primarily 15q21 and 6p21.3) in dyslexia ${ }^{5-12}$. In addition, a genome search in a single Norwegian family has mapped an apparently monogenic form of this trait to 2 p15-p16 using traditional qualitative linkage analysis ${ }^{13}$. Nevertheless, the vast majority of the genome has until now remained unexplored for QTLs influencing reading disability in the wider population of dyslexics ${ }^{4}$. We therefore screened two large independent sets of nuclear families affected by dyslexia using more than 400 microsatellite markers spaced at approximately $10-\mathrm{cM}$ intervals throughout the genome.

The UK sample consisted of 89 families (195 total sibling pairs), ascertained through a dyslexia clinic on the basis of one severely dyslexic child and reported reading problems in one or more siblings ${ }^{7,14,15}$. The US sample comprised 119 families (180 total sibling pairs) drawn from the Colorado twin study of reading disability $^{2}$. In this epidemiological sample, twin-pairs in which at least one member had a positive school history of reading difficulty were systematically recruited from school districts ${ }^{5,6,8}$. For both the UK and US samples, we assessed all the available children in each family, irrespective of a diagnosis of dyslexia, with standardized tests of single-word reading and spelling, as well as mea- sures of hypothesized components of the reading process, including phoneme awareness (PA), phonological decoding (PD) and orthographic coding (OC; Methods) ${ }^{15-17}$. Dyslexics usually show deficits in one or more of these component measures ${ }^{3}$. Because of practical constraints, the specific tests that were administered in the UK and US samples differed, but they tapped comparable skills. All of the measures have been shown to have significant familiality ${ }^{15}$ and/or heritability ${ }^{16}$ in these samples. The inter-trait correlations in each data set are moderate to high, ranging from 0.41 to 0.84 (Web Table A). Recent twin studies have established that a significant proportion (approximately 39-99\%) of the covariance between these reading-related measures is influenced by genetic factors (Web Table B) $)^{16,17}$.

We evaluated the linkage of autosomal genotype data to quantitative measures from each scan using two QTL-based statistical approaches. First, we used maximum-likelihood estimation of variance components (VC) to construct a powerful test of linkage that exploited most of the available trait information ${ }^{18}$. Because this method can be sensitive to deviations from multivariate normality ${ }^{19}$, we adopted a simulation strategy to assess the empirical significance of the results (Fig. 1; see Methods). Second, we used a modified DeFries-Fulker (DF) regression technique, which was originally developed in previous studies on the US sample ${ }^{5}$, and again carried out simulations to evaluate the empirical significance of linkage. The DF approach is expected to be more powerful and appropriate than basic VC methods for analyzing the US sample, as it is designed specifically to take advantage of singleproband ascertainment schemes.

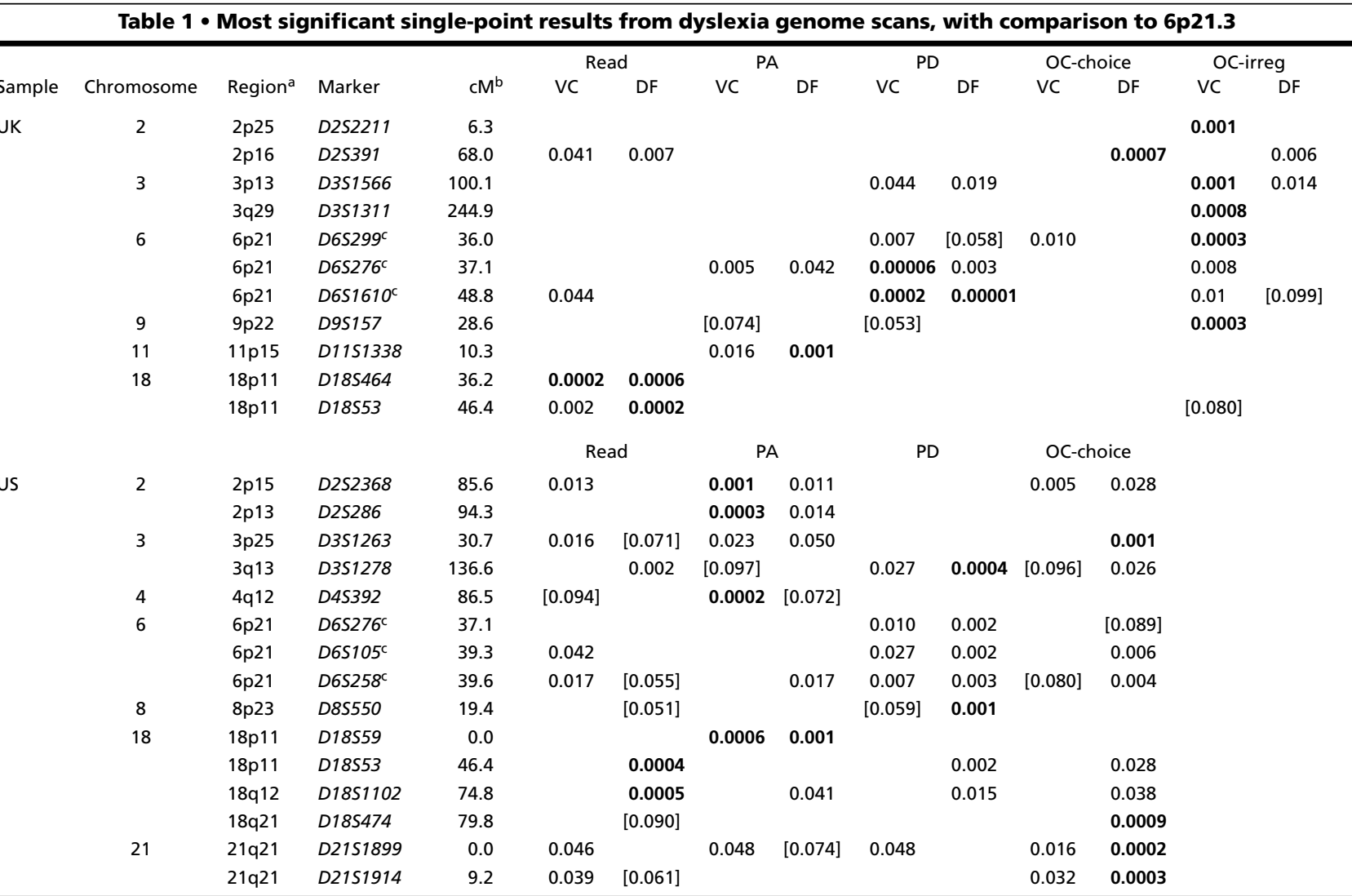

Most significant single-point linkage results from the entire genome in the UK and US samples. For abbreviations, see text. This table shows only those genomescan markers where a nominal $P$ value of 0.001 or less was given by at least one measure with one analytical method (bold type). Any $P$ values that were 0.1 or less with other trait/method combinations are also given for this selection of markers. $P$ values between 0.05 and 0.1 are shown in square brackets. aMost likely

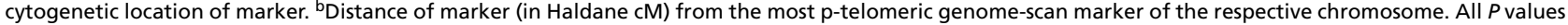
were greater than 0.05 for analyses of the spelling phenotype with these markers. 'For comparison, we also show the three most significant markers from targeted analysis of $6 p$ in each sample; this investigation incorporated genotype data from previous studies of $6 p^{5-8}$. Each of the most significant $6 p$ markers in the UK sample yielded $P$ values of 0.001 or less. In contrast, all $6 \mathrm{p}$ markers in the US sample gave $P$ values of greater than 0.001 . (See also Web Tables B and C.) 
We have previously used subsets of both genome-screen family collections to investigate the $6 \mathrm{p} 21.3$ region $^{5-8}$. QTL analyses of chromosome $6 \mathrm{p}$ markers in the genome-screen samples were consistent with previous findings, which indicated that a locus in the vicinity of D6S276-D6S105 influenced phonological and orthographic processing (Table 1). For the first time, however, we have been able to assess the evidence supporting the role of the $6 \mathrm{p} 21.3$ QTL in the context of linkage data across the entire genome (Tables 1 and 2). For the UK sample, 6p21.3 markers did indeed provide some of the most significant results, although a number of regions on other chromosomes (including 2, 3, 9 and 18) were also highlighted (Web Table $\mathrm{C}$ and Web Figs $\mathrm{A}$ and $\mathrm{B}$ ). By contrast, in the US sample the chromosome 6p QTL did not stand out dramatically against the genomic background; linkage evidence was more substantial for several other regions, particularly on chromosomes 2, 3, 18 and 21 (Web Table D and Web Figs C and D).

In single-point analysis of each genome scan, the most significant result for single-word reading came from the same marker, $D 18 S 53$, with $P=0.0002$ (UK-DF) and $P=0.0004$ (US-DF). The strongest multipoint linkage peak in the entire UK scan lay between D18S464 and D18S53 in 18p11.2; the evidence from VC analyses of single-word reading (pointwise empirical $P$ value $=0.00001)$ exceeded the conservative threshold $(0.00002)$ for genome-wide significance put forward by Lander and Kruglyak (Fig. 2a) ${ }^{20}$. The empirical $P$ value still approached this threshold after conservatively accounting for the testing of multiple measures $(P \leq 0.00006)$. The same region of $18 \mathrm{p} 11.2$ yielded some of the strongest multipoint results in the US sample (Table 2), with highest significance for the DF analysis of single-word reading (empirical $P=0.0004$; Fig. $2 a$ ). Measures of phonological and orthographic coding were also linked to the 18p11.2 QTL in both screens, but the evidence in the UK sample for these traits was somewhat weaker (Table 2). It is also worth noting that there was in the US sample a second clear peak, mapping approximately $80 \mathrm{cM}$ from the first, on the opposite arm of chromosome 18.

Another region that was independently highlighted by both the UK and US genome screens was 2p15-p16, although the multipoint peak in the UK sample was weaker than and more distal to that found in the US group. The position of the 2p15 QTL in the US sample overlapped with the region identified by previous qualitative analysis of a large Norwegian pedigree ${ }^{13}$. Our findings thus indicate that, rather than being restricted to rare cases of apparent monogenic inheritance, the 2p15-p16 locus is likely to have a more general role as a QTL for reading disability in a wider population. Multipoint QTL analyses of X-linked markers using the traditional Haseman-Elston (HE) approach (Methods) ${ }^{21,22}$ also suggested a locus on Xq26 (empirical $P=0.001$ ) in the UK sample (Web Fig. E), which is notable given the possibility that there may be a higher incidence of dyslexia in males than in females ${ }^{23}$.

In the absence of any clear consensus regarding the nature of the core deficits in this neurological syndrome, we adopted the strategy of analyzing multiple reading-related measures to maximize our potential to detect QTLs influencing dyslexia. In addition, twin studies have shown that genetic factors contribute not only to the $a$

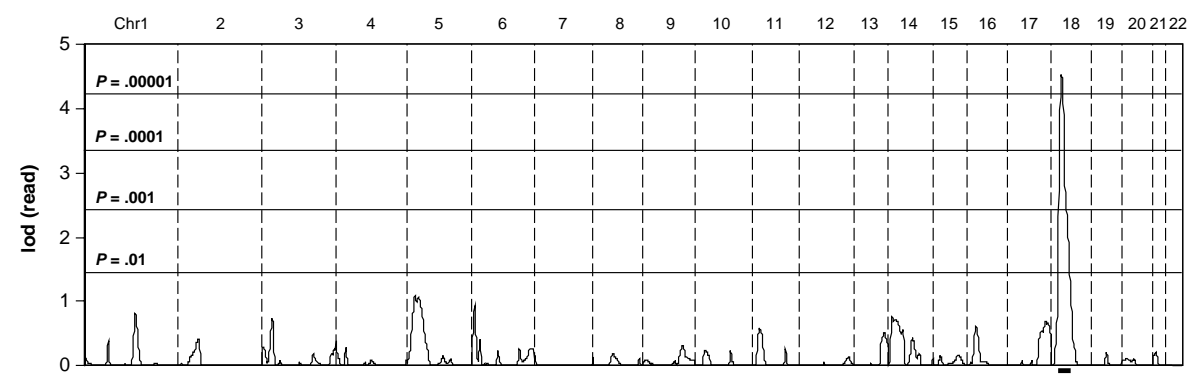

US - DF

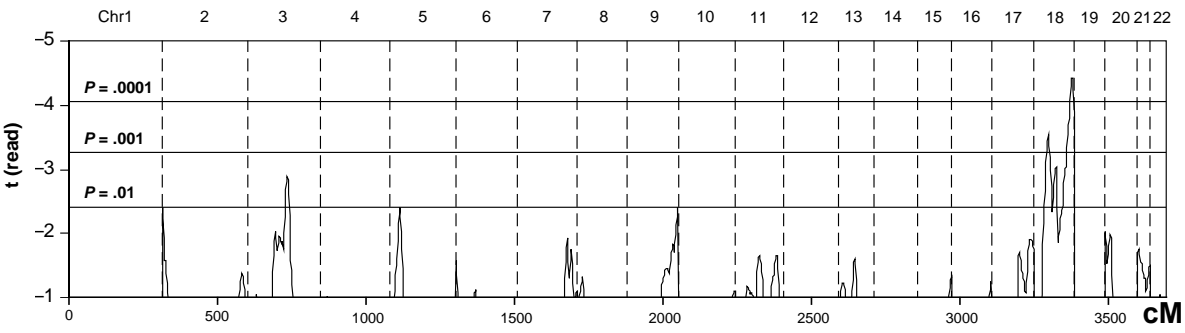

$b$

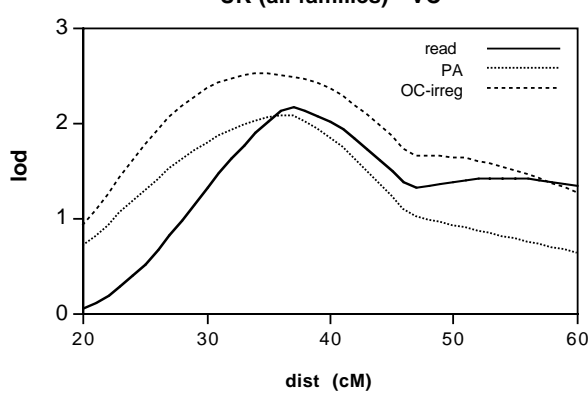

US - DF

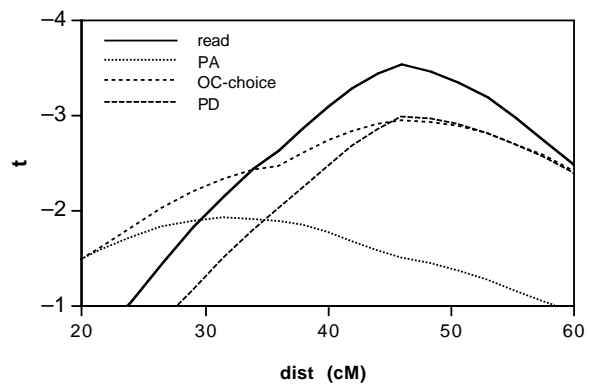

Fig. 2 A new QTL on 18p influences dyslexia. $a$, Multipoint linkage analysis of single-word reading in the UK and US genome-screen samples shows concordant evidence for an $18 \mathrm{p}$ QTL. The more appropriate analytical method for each sample is shown: VC for UK and DF for US. The latter method takes advantage of the single-proband ascertainment scheme of the US sample, yielding increased power. Analyses of all trait/method combinations (Table 2 and Web Figs A-E) indicate a number of additional loci that may also influence developmental dyslexia. Measure- and sample-specific empirically derived $P$ value thresholds are superimposed on the curves. The cumulative distance (in Haldane $\mathrm{CM}$ ) is displayed along the bottom, with chromosome numbers at the top. The thick line beneath the UK-VC plot indicates the 3-lod unit support interval for the $18 p$ locus indicated by this analysis, spanning approximately $40 \mathrm{cM}$ and containing markers D185464 and D18553. $\boldsymbol{b}$, The 18p QTL influences several measures related to reading in the UK and US samples. The region shown here corresponds to the 3-lod unit support interval indicated in $a$. The combined VC analyses of all UK families (including genome-screen and replication set) yielded linkage to the Read, PA and OC-irreg measures. Empirically derived $P$ values for these results are all less than or equal to 0.003 . The DF analyses of US genome-screen families gave empirical $P$ values that were all less than or equal to 0.005 for Read, PD and OC-choice measures in the same interval. 
Table $2 \cdot$ Most significant multipoint results from dyslexia genome scans

\begin{tabular}{|c|c|c|c|c|c|c|c|c|c|c|}
\hline \multirow[b]{2}{*}{ Sample } & \multirow[b]{2}{*}{ Region $^{c}$} & & \multicolumn{2}{|c|}{ Read } & \multicolumn{2}{|c|}{ PA } & \multicolumn{2}{|c|}{ PD } & \multicolumn{2}{|c|}{ OC-irreg } \\
\hline & & & VC & DF & VC & DF & VC & DF & VC & DF \\
\hline \multirow[t]{8}{*}{ UK } & $6 p 21$ & $P$ value ${ }^{a}$ & 0.031 & [0.062] & 0.008 & [0.054] & 0.001 & 0.002 & 0.022 & [0.058] \\
\hline & & position ${ }^{b}$ & 6 & 6 & 29 & 18 & 37 & 27 & 38 & 22 \\
\hline & $18 p 11$ & $P$ value ${ }^{a}$ & 0.00001 & 0.0002 & & & [0.073] & & 0.012 & [0.064] \\
\hline & & position $^{b}$ & 34 & 40 & & & [38] & & 31 & [40] \\
\hline & & & $\mathrm{HE}$ & & & & $\mathrm{HE}$ & & $\mathrm{HE}$ & \\
\hline & Xq26 & $P$ value ${ }^{a}$ & 0.001 & & & & 0.018 & & 0.038 & \\
\hline & & position $^{b}$ & 168 & & & & 169 & & 169 & \\
\hline & & & \multicolumn{2}{|c|}{ Read } & \multicolumn{2}{|c|}{ PA } & \multicolumn{2}{|c|}{ PD } & \multicolumn{2}{|c|}{ OC-choice } \\
\hline \multirow[t]{11}{*}{ US } & $2 p 15$ & $P$ value ${ }^{a}$ & 0.006 & & 0.002 & 0.001 & & & 0.005 & 0.007 \\
\hline & & position $^{b}$ & 85 & & 86 & 83 & & & 83 & 81 \\
\hline & $3 q 13$ & $P$ value ${ }^{\mathrm{a}}$ & 0.017 & 0.003 & & [0.072] & 0.046 & 0.0003 & & 0.025 \\
\hline & & position $^{b}$ & 90 & 136 & & [144] & 134 & 136 & & 131 \\
\hline & $13 q 22$ & $P$ value & 0.039 & & & & 0.014 & & 0.001 & [0.053] \\
\hline & $18 p 11$ & $P$ value ${ }^{a}$ & & 0.0004 & 0.004 & 0.006 & & 0.003 & & 0.005 \\
\hline & & position $^{b}$ & & 46 & 0 & 4 & & 46 & & 46 \\
\hline & $18 q 22$ & $P$ value ${ }^{a}$ & 0.021 & 0.00003 & & & 0.012 & 0.001 & 0.027 & 0.0009 \\
\hline & & position $^{b}$ & 122 & 127 & & & 128 & 127 & 119 & 101 \\
\hline & $21 q 21-q 22$ & $P$ value ${ }^{a}$ & {$[0.052]$} & 0.045 & & & 0.024 & 0.026 & 0.004 & 0.00003 \\
\hline & & position $^{b}$ & [1] & 2 & & & 36 & 42 & 28 & 4 \\
\hline
\end{tabular}

Most significant multipoint linkage results from the entire genome in the UK and US samples. apointwise $P$ values are reported, all of which were empirically derived via simulations as outlined in Methods. This table only includes regions where a peak $P$ value $(\leq 0.001)$ was found for at least one measure with VC or DF analysis (or HE analysis in the case of the $\mathrm{X}$ chromosome; see text). These $P$ values are displayed in bold type. ${ }^{b}$ The position of peak linkage is given in cM from the most p-telomeric marker of the chromosome. 'Most likely cytogenetic location of the strongest peak. $P$ values and positions of the nearest peaks for other traits/methods are given when $P$ is less than or equal to 0.1 . Square brackets denote $P$ values of between 0.05 and 0.1 . (See also Web Figs A-E.)

correlated variance between such measures ${ }^{16,17}$ but also to the independent variance of different components ${ }^{17,24}$. However, as previously discussed ${ }^{4,7,25}$, although the sample sizes investigated here may be sufficient for the detection of linkage to major QTLs, they are too small to yield reliable estimates of relative effect sizes for each hypothesized reading component at any particular QTL. Factors such as the variable sensitivity of psychometric tests, age difference or stochastic variation between family samples may give rise to spurious variability in linkage evidence for alternative measures at a QTL of interest ${ }^{4,7,25}$. The 18p11.2 QTL identified in the present study influenced several measures at similar levels in the US sample, but in the UK genome-screen sample the linkage evidence came predominantly from single-word reading. We therefore proceeded to genotype five of the genome-screen markers spanning the region of interest in a new set of 84 UK families (143 total sibling pairs), providing us with a significantly larger total UK sample with which to explore the phenotypic profile influenced by this QTL.

Independent analysis of the new families again showed strong evidence for the involvement of the 18p11.2 QTL, but in this case the PA phenotype gave the most significant results, with $P$ values of less than 0.0005 adjacent to D18S464 (Table 3). The influence of the 18p11.2 QTL on the reading measures did not seem to differ substantially between the two sets of UK families (likelihoodratio test of heterogeneity, $P>0.02$ for each linked marker). In addition, twin studies have previously indicated a high genetic correlation (approximately 0.7) between measures of singleword reading and $\mathrm{PA}^{17}$. A combined analysis of all the UK families (including 338 total sibling pairs) supported the view that the 18p11.2 QTL does indeed influence multiple components of the reading process, as suggested from the US sample (Fig. $2 b$ ). Nevertheless, investigation of 18p11.2 in much larger data sets is needed to obtain a precise estimate of its relative influence on specific reading-related processes. We are in the process of developing fully multivariate methods to address this issue further.

In conclusion, our current findings continue to support previous suggestions of a role for $6 \mathrm{p} 21.3$ in reading disability (the majority of evidence for which came from subsets of the samples studied here ${ }^{5-8}$ ) but also point to a number of other potential loci of interest. In particular, they indicate that a newly identified QTL on 18p11.2 makes a much more significant contribution to

\begin{tabular}{|c|c|c|c|c|c|c|c|c|c|c|c|}
\hline \multirow[t]{2}{*}{ Single-point } & \multirow[b]{2}{*}{$\mathrm{CM}^{\mathrm{b}}$} & \multicolumn{2}{|c|}{ Read } & \multicolumn{2}{|c|}{ PA } & \multicolumn{2}{|c|}{ PD } & \multicolumn{2}{|c|}{ OC-irreg } & \multicolumn{2}{|c|}{ Spell } \\
\hline & & VC & DF & VC & DF & VC & DF & VC & DF & VC & DF \\
\hline D18S464 & 36.2 & [0.088] & [0.062] & 0.0001 & 0.00004 & & & 0.015 & 0.002 & & 0.011 \\
\hline D18S53 & 46.4 & & & 0.003 & 0.002 & & & 0.017 & & & [0.098] \\
\hline D18S478 & 62.3 & & & & & & & & & & \\
\hline \multicolumn{12}{|l|}{ Multipoint } \\
\hline \multirow[t]{2}{*}{$18 p 11$} & $P$ value ${ }^{a}$ & 0.016 & {$[0.080]$} & 0.0005 & 0.001 & 0.042 & & 0.012 & 0.043 & & 0.048 \\
\hline & position ${ }^{b}$ & 75 & [75] & 36 & 36 & 75 & & 37 & 36 & & 37 \\
\hline
\end{tabular}


dyslexia susceptibility. This QTL was robustly identified in three large independent samples with a level of concordance rare for complex phenotypes. Our study thus demonstrates the value of using a QTL-based genome-wide search strategy when investigating complex cognitive traits.

\section{Methods}

Ascertainment of UK sample. We identified all the UK families through the dyslexia clinic at the Royal Berkshire Hospital, Reading7,15. We selected children whose single-word reading was more than 2 s.d. below that predicted by tests of verbal or nonverbal reasoning, as described ${ }^{7,15}$. We included families in the study if there was also evidence of reading disability in one or more siblings of the dyslexic child (for example, on the basis of parental reports or school history $)^{7,15}$. The genome-screen sample comprised 89 such families, many of which contained multiple sibships, yielding 135 independent or 195 total sibling pairs for linkage analysis. This includes 82 families from a previous report that evaluated $6 \mathrm{p} 21.3$ linkage to reading-related measures using the HE and VC methods ${ }^{7}$. The sample collected for 18p11 replication comprised 84 new families (none of which had been included in the genomescreen sample) containing 112 independent or 143 total sibling pairs.

Ascertainment of US sample. The US genome-screen sample comprised 119 families taken from the Colorado twin study of reading disability ${ }^{2}$. For the latter, we identified twin-pairs from the records of 27 Colorado schoo districts and selected those in which at least one member had a positive school history of reading difficulty $2,5,6,8$. The sample investigated in the current report also included non-twin siblings in a significant proportion of families, yielding 147 independent or 180 total sibling pairs for linkage analysis. We did not use pairs of monozygotic twins in this study. Of the 119 families analyzed in the scan, 39 came from the original study that had first suggested 6p21.3 linkage to a composite index of dyslexia severity ${ }^{5,6}$, and another 70 came from a follow-up study examining the contribution of specific reading and language skills ${ }^{8}$; both these studies used a DF-based approach for investigating linkage.

Phenotypic measures. For each sample, we administered a battery of psychometric tests to all the available children in each family and standardized thei scores against normal controls, as described in previous publications ${ }^{8,15-17}$ We originally collected the UK and US sets of families in separate independent studies; as a consequence, the UK and US batteries differed with respect to the specific tests that were included, but these assessed comparable skills. Measures in both samples evaluated single-word reading, spelling and a series of skills that have been held to be components of the reading process.

'Phoneme awareness' is defined as the capacity to reflect explicitly on the individual speech sounds that make up a word (phonemes). We assessed this ability using oral tasks that did not involve any visual processing of print, such as the 'spoonerism' test (UK sample) ${ }^{15}$ and tests of phoneme transposition/deletion (US sample) ${ }^{8}$. We measured 'phonological decoding, the ability to convert written sublexical letter units (graphemes) into their corresponding phonemes, through the oral reading of pronounceable non-words that lack real meaning (such as torlep) ${ }^{7,8,15}$. 'Orthographic coding' may be defined as the ability to recognize the specific letter pattern (orthography) of whole words. We assessed this using forced-choice tasks (OC-choice) that required rapid recognition of a target word compared with a phonologically identical non-word (such as rain versus rane) $)^{8,15-17}$ A second test of OC (OC-irreg), which we used only in the UK study, measured success in the oral reading of irregular words (such as yacht and colonel) that violate the standard letter-sound conventions of English ${ }^{7,15}$. Such words cannot be read accurately by grapheme-phoneme conversion rules, so this test should place greater emphasis on orthographic processing than on PD. A statistical exploration of test scores from the UK sample has indicated that all these reading-related measures are significantly influenced by familial factors and have sufficient variance for a QTL-based mapping study ${ }^{15}$. Extensive studies of the Colorado twin sample from which the US sample was drawn indicated that the measures are significantly heritable ${ }^{16,17,24}$. Inter-trait correlations are given in Web Table A.

Genome screens. We genotyped 401 highly polymorphic markers, spanning all 22 autosomes and the X chromosome, in children and parents from each family. Thirty-nine US families included only one parent; the remaining 80 US families and all 89 UK families included both parents. The majority of markers came from the ABI PRISM LMS2-MD10 panels (Applied Biosystems). We took sex-averaged marker maps from CHLC (Co-operative Human Linkage Center) supplemented with data from Généthon ${ }^{26}$, and verified these by comparison with maps estimated from our genome-screen marker data. We carried out semi-automated fluorescent genotyping using standard techniques as described ${ }^{7}$. We checked raw allele size data for consistent inheritance and converted them to LINKAGE format using the GAS software package (version 2.0; Alan Young, Oxford University). We used Discovery Manager (Genomica Corporation) for the storage of genotypic and phenotypic data and the creation of the appropriate files for statistical analysis. As a further check on genotyping quality, we generated marker haplotypes from the data using Genehunter2.0 (GH2.0; ref. 27) to identify any chromosomes showing an excessive number of recombination events. The statistical analyses also incorporated genotypic data, from 16 markers in the UK sample and eight in the US sample, which had previously been generated for targeted studies of the 6p21.3 QTL ${ }^{5-8}$.

Variance components linkage analysis. We carried out VC linkage analysis with GH2.0 (ref. 27), estimating a mean and with no dominance VCs. The method involves maximum-likelihood estimation of major gene $\left(\sigma^{2} \mathrm{a}\right)$, polygenic $\left(\sigma^{2} \mathrm{~g}\right)$ and environmental $\left(\sigma^{2} \mathrm{e}\right)$ VCs contributing to trait variability between siblings for each locus under investigation ${ }^{18}$. The likelihood assuming a major gene effect at this locus is compared with that under the null hypothesis of no major gene effect (when $\sigma^{2}$ a is constrained to be zero). The lod scores output by GH2.0 could be converted into nominal $P$ values by multiplying by $2 \log _{e} 10$ and then determining significance from $\chi^{2}$ tables, as described ${ }^{20}$. We included all siblings for VC analysis.

DeFries-Fulker linkage analysis. We used identity-by-descent (IBD) probability distributions, exported from GH2.0, as input for basic DF linkage analysis, which employed QTL macros written for the SAS package ${ }^{28}$. The DF linkage approach involves designating probands who score below an arbitrary predefined threshold for each measure (relative to a control population) and assessing whether their co-sibs regress to the population mean as a function of $\mathrm{IBD}^{5,8}$. In a large sample, the significance of this relationship can be estimated from a $t$-test of the appropriate regression coefficient ${ }^{5}$. Before conducting QTL analysis, we decided on thresholds of -1 s.d. for the UK sample ${ }^{15}$ and -2 s.d. for the US sample ${ }^{8}$, which maintained sufficiently large sample sizes to provide robust $t$-tests for linkage. We conducted the analysis with only a single threshold in each sample in order to minimize multiple testing. The numbers of proband/co-sib pairs for multipoint DF analysis for each measure were: UK (genome screen), Read 115, Spell 168, PA 90, PD 82, OC-choice 110, OC-irreg 140; UK (replication), Read 78, Spell 91, PA 40, PD 54, OC-choice 91, OC-irreg 89; US, Read 137, PA 71, PD 103, OC-choice 72. The test accommodated multiple sibships by employing reduced degrees of freedom corresponding to the number of unique pairings. For families in which more than one proband had been defined, the proband-proband pairings were double-entered, but the degrees of freedom were adjusted accordingly ${ }^{28}$.

X-linked QTL analysis. VC/DF analysis of X-linked data is not yet fully implemented. We therefore carried out the analysis of X-linked markers using the more traditional HE method ${ }^{21}$, which regresses sibling-pair squared trait differences against IBD sharing, as implemented in the MAPMAKER/SIBS ${ }^{22}$ software package. For X-linked data, MAPMAKER/SIBS uses all siblings to determine maternal phase/allele information but considers only phenotyped males when assessing HE linkage. We used the 'all-pairs, unweighted' option to handle multiple sibships, and estimated $P$ values using simulation. The results from the $\mathrm{HE}$ analyses of autosomal data agreed well with those from $\mathrm{VC}$ analyses, implicating the same regions (data not shown).

Simulations. The results of the VC test may be influenced by deviations from multivariate normality ${ }^{19}$. We evaluated this for the various measures of each sample as follows. We used the program SIMULATE (http://linkage.rockefeller.edu/ott/) to simulate a single unlinked marker with four equally frequent alleles (75\% heterozygosity), while maintaining the family structures and phenotypic scores of the sample (that is assuming the null hypothesis of no linkage between genotype and phenotype). We ran 100,000 replicates and analyzed data from each for QTL linkage using the VC approach. We postulated that the resulting 
lod-significance distributions (Fig. 1) would approximate that found at each point of a typical multipoint situation (from which an average of $70-80 \%$ of IBD information is extracted ${ }^{22}$ ) and should therefore be generally applicable for estimating the pointwise significance of linkage peaks.

To verify this, we specifically investigated the chromosome 18 single word-reading linkage in the UK genome-screen sample. We simulated the entire chromosome 100,000 times according to the real-data allelic numbers and frequencies, patterns of missing genotype data and genetic distances between markers (again assuming no genotype-phenotype linkage). We then assessed VC linkage at the location that had shown peak single-wordreading linkage with the real genotype data. The resulting lod-significance distribution was effectively identical to the corresponding single-marker situation, showing not only that the $18 \mathrm{p}$ locus had no unusual marker/map properties, but also that the single-marker simulation approach was likely to be generally applicable. Although more robust than VC methods, estimates of extreme $P$ values from regression-based methods may also be sensitive to assumptions. We therefore also analyzed 100,000 replicates of each sample with the DF approach, using the resulting $t$-test-significance distributions for the estimation of empirical pointwise $P$ values. Finally, we carried out 100,000 simulations of a dummy X-chromosome marker with four equally frequent alleles and analyzed them with the 'all-pairs, unweighted' option of MAPMAKER/SIBS ${ }^{22}$, allowing us to adjust for any inflation of significance that might result from the statistical dependence of multiple sibships.

All empirical $P$ values, although adjusted to account for measure-specific deviations from normality or the statistical dependence of multiple sibships, still yielded only pointwise estimates of significance; they were not adjusted to account for the scanning of the whole genome. In addition, although the approximate magnitudes of extreme $P$ values $(<0.0001)$ are likely to be estimated correctly using 100,000 simulations, their precise specification is not possible without performing millions of simulations, which is impractical.

Heterogeneity test. To test for heterogeneity of $18 \mathrm{p}$ linkage between the two separate sets of UK families, we performed a VC-based likelihoodratio test for each linked marker. We first standardized measures within each set of families. Given an unconstrained $\sigma^{2} \mathrm{a}$, if $\mathrm{LL}_{1}$ is the maximized $\log$-likelihood of the data for the genome screen families, $\mathrm{LL}_{2}$ is that for the replication set and $\mathrm{LL}_{C}$ is that for a combined analysis of all families, then:

$$
\mathrm{LRT}=2 \times\left(\mathrm{LL}_{1}+\mathrm{LL}_{2}-\mathrm{LL}_{\mathrm{C}}\right),
$$

which is distributed as a $\chi^{2}$ statistic with 1 degree of freedom.

Note: Supplementary information is available on the Nature Genetics web site (http://genetics.nature.com/supplementary_info/).

\section{Acknowledgments}

We are very grateful to all the families who participated in this study. We thank J. Walter, P. Southcott, S. Fowler and C. Clisby for collection of the UK families; K. Taylor for assistance with handling the UK phenotype data; $H$. Rees, J. Smith and P. Kelley for assistance with genotyping; and D. Weeks and S. Cherny for advice on statistical analyses. Collection of the UK families and all genotyping were funded by the Wellcome Trust. The US study was supported in part by grants from the National Institute of Child Health and Human Development and the National Institute of Mental Health. L.R.C. was funded in part by an NIH grant. I.L.M is funded by the British Council and NSERC (Canada). C.F. was a Wellcome Trust Prize Student. A.P.M. is a Wellcome Trust Principal Research Fellow.

\section{Received 21 August; accepted 1 November 2001.}

1. Brown, B.S. in Dyslexia: An Appraisal of Current Knowledge (eds Benton, A.L. \& Pearl, D.) v-vii (Oxford University Press, Oxford, 1978).

2. Defries, J.C., Fulker, D.W. \& LaBuda, M.C. Evidence for a genetic aetiology in reading disability of twins. Nature 329, 537-539 (1987).

3. Habib, M. The neurological basis of developmental dyslexia. An overview and working hypothesis. Brain 123, 2373-2399 (2000).

4. Fisher, S.E. \& Smith, S.D. in Dyslexia: Theory and Good Practice (ed. Fawcett, A.J.) 39-64 (Whurr, London, 2001).

5. Cardon, L.R. et al. Quantitative trait locus for reading disability on chromosome 6. Science 266, 276-279 (1994)

6. Cardon, L.R. et al. Quantitative trait locus for reading disability: correction. Science 268, 1553 (1995)

7. Fisher, S.E. et al. A quantitative trait locus on chromosome $6 \mathrm{p}$ influences different aspects of developmental dyslexia. Am. J. Hum. Genet. 64, 146-156 (1999).

8. Gayán, J. et al. Quantitative trait locus for specific language and reading deficits on chromosome 6p. Am. J. Hum. Genet. 64, 157-164 (1999).

9. Grigorenko, E.L. et al. Susceptibility loci for distinct components of developmental dyslexia on chromosomes 6 and 15. Am. J. Hum. Genet. 60, 27-39 (1997).

10. Field, L.L. \& Kaplan, B.J. Absence of linkage of phonological coding dyslexia to chromosome 6p23-p21.3 in a large family data set. Am. J. Hum. Genet. 63, 1448-1456 (1998).

11. Schulte-Körne, G. et al. Evidence for linkage of spelling disability to chromosome 15. Am. J. Hum. Genet. 63, 279-282 (1998).

12. Morris, D.W. et al. Family-based association mapping provides evidence for a gene for reading disability on chromosome 15q. Hum. Mol. Genet. 9, 843-848 (2000).

13. Fagerheim, T. et al. A new gene (DYX3) for dyslexia is located on chromosome 2 . J. Med. Genet. 36, 664-669 (1999).

14. Fisher, S.E., Stein, J.F. \& Monaco, A.P. A genome-wide search strategy for identifying quantitative trait loci involved in reading and spelling disability (developmental dyslexia). Eur. Child Adolesc. Psych. 8, 47-51 (1999).

15. Marlow, A.J. et al. Investigation of quantitative measures related to reading disability in a large sample of sib-pairs from the UK. Behav. Genet. 31, 219-230 (2001).

16. Olson, R.K., Forsberg, H. \& Wise, B. in The Varieties of Orthographic Knowledge Vol I: Theoretical and Developmental Issues (ed. Berninger, V.W.) 27-71 (Kluwer Academic, Dordrecht, 1994).

17. Gayán, J. \& Olson, R.K. Genetic and environmental influences on orthographic and phonological skills in children with reading disabilities. Dev. Neuropsych. 20 487-511 (2001).

18. Amos, C.I. Robust variance-components approach for assessing genetic linkage in pedigrees. Am. J. Hum. Genet. 54, 535-543 (1994).

19. Allison, D.B. et al. Testing the robustness of the likelihood-ratio test in a variancecomponent quantitative-trait loci-mapping procedure. Am. J. Hum. Genet. 65, 531-544 (1999).

20. Lander, E. \& Kruglyak, L. Genetic dissection of complex traits: guidelines for interpreting and reporting linkage results. Nature Genet. 11, 241-247 (1995).

21. Haseman, J.K. \& Elston, R.C. The investigation of linkage between a quantitative trait and a marker locus. Behav. Genet. 2, 3-19 (1972).

22. Kruglyak, L. \& Lander, E.S. Complete multipoint sib-pair analysis of qualitative and quantitative traits. Am. J. Hum. Genet. 57, 439-454 (1995).

23. Flannery, K.A., Liederman, J., Daly, L. \& Schultz, J. Male prevalence for reading disability is found in a large sample of black and white children free from ascertainment bias. J. Int. Neuropsychol. Soc. 6, 433-442 (2000).

24. Olson, R.K., Datta, H., Gayán, J. \& DeFries, J.C. in Converging Methods for Understanding Reading and Dyslexia (eds Klein, R.M. \& McMullen, P.A.) 133-151 (MIT Press, Cambridge, MA, 1999).

25. Pennington, B.F. Using genetics to dissect cognition. Am. J. Hum. Genet. 60, 13-16 (1997).

26. Dib, C. et al. A comprehensive genetic map of the human genome based on 5,264 microsatellites. Nature 380, 152-154 (1996)

27. Pratt, S.C., Daly, M.J. \& Kruglyak, L. Exact multipoint quantitative-trait linkage analysis in pedigrees by variance components. Am. J. Hum. Genet. 66, 1153-1157 (2000).

28. Lessem, J.M. \& Cherny, S.C. DeFries-Fulker multiple regression of sibship QTL data: a SAS macro. Bioinformatics 17, 371-372 (2001). 